Guedes, R. M., Pereira, C. M. C., Fonseca, A., \& Oliveira, M. S. A. (2013). Composite Structures, 105, 263-268.

\title{
The effect of carbon nanotubes on viscoelastic behaviour of biomedical grade ultra-high molecular weight polyethylene
}

\author{
Rui Miranda Guedes ${ }^{1,2}$, Celeste Pereira ${ }^{1}$, Alexandra Fonseca ${ }^{3}$ and Mónica S.A. \\ Oliveira $^{3}$ \\ ${ }^{1}$ Instituto de Engenharia Mecânica e Gestão Industrial (INEGI) \\ Rua Dr. Roberto Frias, 4200-465 Porto, Portugal \\ e-mail: cpereira@inegi.up.pt, web page: http://www.inegi.pt \\ ${ }^{2}$ Faculdade de Engenharia da Universidade do Porto (FEUP) \\ Rua Dr. Roberto Frias, 4200-465 Porto, Portugal \\ e-mail: rmguedes@fe.up.pt, web page: http://www.fe.up.pt \\ ${ }^{3}$ Departmento de Engenharia Mecânica, Universidade de Aveiro \\ Campus Universitário de Santiago, 3810-193 Aveiro, Portugal \\ e-mail: monica.oliveira@ua.pt, web page: http://www.ua.pt
}

\begin{abstract}
Nanocomposites of ultra-high molecular weight polyethylene reinforced with multiwalled carbon nanotubes (UHMWPE/MWCNT) have been prepared with different volume fractions of MWCNTs: $0.2,0.4,0.6,0.8$ and 1.0\%. DMTA experiments were carried out using a TA Instruments Q800 equipment and the samples were cut from the compressed sheets of polymer and composites. The experiments were conducted on each sample at 12 different frequencies varying from $0.1 \mathrm{~Hz}$ to $100 \mathrm{~Hz}$ over the temperature range of $22^{\circ} \mathrm{C}-82^{\circ} \mathrm{C}$ at an interval of $5^{\circ} \mathrm{C}$ where the reference temperature was kept at $37^{\circ} \mathrm{C}$. It was verified that both horizontal and vertical shifts are necessary to superimpose the dynamic modulus/frequency curves of all cases. The MWCNT did not seem to change the viscoelastic nature of the UHMWPE, i. e. incorporation of up to $1 \mathrm{wt} . \%$ of MWCNTs has negligible influence on the viscoelastic behaviour of the UHMWPE. Hence the same analytical model is applicable for the viscoelastic description of the nanocomposites.
\end{abstract}

Keywords: Ultra-high molecular weight polyethylene (UHMWPE); Multiwalled carbon nanotube (MWCNT); Viscoelastic properties; Dynamic Mechanical and Thermal Analysis (DMTA); TimeTemperature Superposition Principle (TTSP)

\section{AUTHORS CORRESPONDENCE}

Rui Miranda Guedes

DEMec, Faculdade de Engenharia da Universidade do Porto, Rua Dr. Roberto Frias s/n, 4200-465 Porto, Portugal

Tel: +351 965487084, e-mail: rmguedes@fe.up.pt 


\section{Introduction}

Ultra-high molecular weight polyethylene (UHMWPE) is still considered the key material in total joint arthroplasties. The UHMWPE was introduced for joint arthroplasties more than forty years ago but remains to be a gold standard as an articulating counterface for arthroplasties. Three fundamental aspects contribute for achieving excellent long-term results: superior wear resistance, high fracture toughness and biocompatibility [1-2]. Recently carbon nanotubes (CNTs) have been added to UHMWPE to improve its tribological properties [3]. Yet the mechanical properties, i.e. modulus and strength, are not always enhanced by adding the CNTs. In some cases the observed increase in mechanical performance with the addition of CNTs goes up to a critical content to decrease above this value [4].

Experienced problems such as osteolysis have been reported while using UHMWPE made acetabular cups in the total hip replacement. Reinforcing of UHMWPE with CNTs is one possible way for improving the toughness of the polymer and decreasing the wear debris produced [5].

Nanocomposites (UHMWPE/MWCNT) have been prepared with different volume fractions of multiwalled carbon nanotubes (MWCNTs): 0.2, 0.4, 0.6, 0.8 and $1.0 \%$. The specimens were produced by compression moulding. Dynamic Mechanical and Thermal Analysis (DMTA) were carried out using a TA Instruments Q800 equipment and the samples were cut from the compressed sheets of polymer and composites. The experiments were conducted on each sample at 12 different frequencies varying from $0.1 \mathrm{~Hz}$ to $100 \mathrm{~Hz}$ over the temperature range of $22^{\circ} \mathrm{C}-82^{\circ} \mathrm{C}$ at an interval of $5^{\circ} \mathrm{C}$.

The applicability of the Time-Temperature Superposition Principle (TTSP) to the dynamic viscoelastic properties was checked; both horizontal and vertical shifts were necessary to superimpose the dynamic compliance/frequency curves. A methodology proposed previously [6] was used to determine the horizontal and vertical shifts factors for the TTSP. The DMTA data analysis based on a viscoelastic model [6] proved to be effective when analyzing the mechanical performance of UHMWPE/MWCNT composites.

Since the reason for adding MWCNT to the UHMWPE is to improve its toughness and its wear resistance, the purpose of this study was to assess the effect of the MWCNTs on the UHMWPE and to ensure that there was no degradation of the viscoelastic properties.

\section{Materials}

The homogeneous mixing of MWCNT and UHMWPE powder was done by mechanical ballmilling. Nanocomposites have been prepared with different volume fractions of MWCNTs: 0.2, 
$0.4,0.6,0.8$ and $1.0 \%$. The specimens were produced by compression moulding. The MWCNTs were supplied by Shenzhen Nanotech Port Co., Ltd., China and their specifications are as follows: diameter $60-100 \mathrm{~nm}$, length $5-15 \mu \mathrm{m}$, density $2.16 \mathrm{~g} / \mathrm{cm}^{3}$ and purity $>95 \%$. The medical grade UHMWPE with the trade name GUR1020 was purchased from Ticona, Inc, Germany. The powder has a density of $0.93 \mathrm{~g} / \mathrm{cm}^{3}$, with a particle mean size of $140 \mu \mathrm{m}$ and an average molecular weight of $3.5 \times 10^{6} \mathrm{~g} / \mathrm{mol}$.

\section{Dynamic Mechanical and Thermal Analysis (DMTA)}

The experiments were carried out using a TA Instruments Q800 equipment and the samples were cut from the compressed sheets of polymer and composites $\left(5 \times 10 \times 2 \mathrm{~mm}^{3}\right)$. The experiments were conducted on each sample at 12 different frequencies ranging from $0.1 \mathrm{~Hz}$ to $100 \mathrm{~Hz}$ over the temperature range of $22^{\circ} \mathrm{C}-82^{\circ} \mathrm{C}$ at an interval of $5^{\circ} \mathrm{C}$ where the reference temperature was kept at $37^{\circ} \mathrm{C}$. The maximum applied force was about $8.0 \mathrm{~N}$ which corresponds to a maximum stress of about $0.80 \mathrm{MPa}$. Only, the averaged data were plotted without error bars in order to present the results clearly.

The tensile creep tests for experimental validation were performed at $37^{\circ} \mathrm{C}$ using the same equipment. The creep tests imposed a constant stress of about $0.60 \mathrm{MPa}$.

\section{Viscoelastic model}

The UHMWPE proved to be a thermorheologically simple material [6], i.e. the distribution trend of the relaxation or retardation times is independent of temperature and the stress magnitudes at all frequencies or times display the same temperature dependency [7].

Short-term experimental measurements at different temperatures are shifted via the TimeTemperature Superposition Principle (TTSP) to those at the reference temperature on a semi-log axis. All curves measured at different temperature levels $S(T)$ collapse into a master curve when plotted as $b_{T} S(T)$ versus $t / a_{T}$ on a log-log scale. The shift factors $a_{T}$ (horizontal) and $b_{T}$ (vertical) are functions of temperature, although the $b_{T}$ dependence is usually weak [7]. If the previous conditions do not hold, the material is classified as thermorheologically complex and all the model parameters become temperature dependent [8].

In a previous work [6] it was verified that both horizontal and vertical shifts, given by $a_{T}$ and $b_{T}$ respectively, were necessary to superimpose the dynamic modulus/frequency curves of pristine UHMWPE. The methodology was based on a viscoelastic model developed to 
determine these shift factors in an easy and effective way [6]. This viscoelastic model was derived from a fractional Maxwell model, which resulted in the creep power law that was successfully applied to represent creep compliance of several polymers [9],

$$
S(t)=b_{T}\left[S_{0}+S_{1} \frac{\left(\frac{t}{a_{T} \tau_{0}}\right)^{\alpha}}{\Gamma(\alpha+1)}\right]
$$

where $S_{0}$ represents the elastic compliance and $S_{1}, \tau_{0}$ and $\alpha$ represent the viscoelastic parameters. This constitutive equation complies with the definition given by Delay and Plazek [7] for thermorheologically simple materials.

The horizontal shift and the reciprocal of the vertical shift are given by the Arrhenius relation as, respectively,

$$
\ln \left(a_{T}\right)=\frac{\Delta H_{a}}{R}\left(\frac{1}{T}-\frac{1}{T_{0}}\right)
$$

and

$$
\ln \left(\frac{1}{b_{T}}\right)=\frac{\Delta H_{b}}{R}\left(\frac{1}{T}-\frac{1}{T_{0}}\right)
$$

where $R$ is the gas constant, $8.314 \mathrm{E}-3 \mathrm{~kJ} /(\mathrm{K} \mathrm{mol}), \Delta H_{a}$ and $\Delta H_{b}$ are the relaxation or retardation activation energies and $T_{0}$ is the reference temperature in ${ }^{\circ} \mathrm{K}$.

In the frequency domain the storage and loss compliance [6] are given by

$$
\begin{aligned}
& S^{\prime}(w)=b_{T}\left[S_{0}+S_{1}\left(\mathrm{w} a_{T} \tau_{0}\right)^{-\alpha} \cos \frac{\alpha \pi}{2}\right], \\
& S^{\prime \prime}(w)=b_{T} S_{1}\left(\mathrm{w} a_{T} \tau_{0}\right)^{-\alpha} \sin \frac{\alpha \pi}{2} .
\end{aligned}
$$

The methodology to determine the elastic-viscoelastic parameters, from DMTA data, is described in detail elsewhere [6]. 


\section{Results and discussion}

The mechanical behaviour of neat UHMWPE and its nanocomposites were studied by measuring the storage compliance and the loss compliance of the sample under sinusoidal load for different temperatures and frequencies. Figure 1 shows the evolution of storage compliance with frequencies varying from $0.1 \mathrm{~Hz}$ to $50 \mathrm{~Hz}$ at $37^{\circ} \mathrm{C}$ for the neat UHMWPE and UHMWPE/MWCNT (1.0\%). The storage compliance decreases towards higher frequency for the neat UHMWPE and UHMWPE/MWCNT (1.0\%). A slight decrease of the nanocomposite storage modulus is observed for all frequencies. However the storage compliance of the neat UHMWPE and UHMWPE/MWCNT (1.0\%) nanocomposite increases with an increase of temperature as shown in Figure 2. A small decrease of the nanocomposite storage compliance is observed for lower temperatures but for higher temperatures the reinforcement effect vanishes. Figures 3-4 show the effect of frequency and temperature on the loss compliance. It is observed that there is not an appreciable difference in loss compliance between neat UHMWPE and UHMWPE/MWCNT (1.0\%) nanocomposite. However a small decrease of the nanocomposite loss compliance is observed for higher temperatures but for lower temperatures the reinforcement effect vanishes. In a previous work done by some of the authors [10], the mechanical behaviour of (High Density Polyethylene) HDPE and HDPE/MWCNT (1\%) nanocomposite were studied by DMTA. The conclusions drawn then were different, since the reinforcing effect of MWCNT was confirmed and it was concluded that the large scale polymer relaxations in the composites were effectively restrained by the presence of MWCNTs. The main explanation for this apparent contradiction may reside on the different productions techniques used which are related with polyethylene grade. The polyethylene classification is based mostly on its density and branching. The molecules of HDPE in general have between 700 to 1800 monomer unites per molecule while UHMWPE molecules have between 100000250000 monomers each [11]. The production of HDPE and HDPE/MWCNT (1\%) nanocomposites specimens used the following technique. The HDPE pallets with a uniform coating of chemically treated nanotubes were melted at the plasticized unit of the injection moulding machine which was kept at $200{ }^{\circ} \mathrm{C}$ to induce sufficient softening of polymer to mix with MWCNTs and this mixture was injected into a tensile specimen. This contrasts with the technique used to produce UHMWPE and UHMWPE/MWCNT nanocomposites. In the present case the specimens were made by compression moulding applied to an homogenous mixture of MWCNT and UHMWPE powder which was obtained by mechanical ball-milling. Therefore it should be expected that the quality of the interface between the polymer and MWCNTs be dependent on the production technique. Therefore it seems that the present technique produces interfaces between the polymer and MWCNTs that are not as effective as those reported previously for the HDPE [10]. 
The model parameters were calculated from the dynamic testing data. In all cases it was verified that the temperature dependency of the exponent parameter $\alpha$ was quite similar, as shown in Figure 5. Moreover the averaged value of $\alpha$ was used for the present analysis, i.e. it was assumed that $\alpha$ was constant. Consequently, the $\alpha$ averaged values for all cases was quite close as it can be depicted from Figure 6 .

Afterwards the other two model parameters were determined, $S_{0}$ and $S_{1}$, for the neat UHMWPE and its nanocomposites. These parameters represent the initial compliance and the coefficient of the time dependent term, respectively. The results are plotted in Figure 7, where it is noticeable a slight decrease of the initial compliance for the UHMWPE/MWCNT $(0.2 \%)$ when compared against the neat UHMWPE and the remaining nanocomposites.

The amount of shifting along the horizontal (x-axis) and vertical (y-axis) in the TTSP plots to align the experimental data points into the master curve was described by the Arrhenius model, given by Equations (2) and (3). The respective activation energies are plotted in Figure 8 for all cases. Again the values are very similar for both shift factors and all cases, implying that the nanoreinforcement did not change the thermal activated processes in the UHMWPE matrix. The storage and loss compliance master curves, for the UHMWPE/MWCNT (1.0\%), can be depicted from Figure 9. All the points obtained at different temperatures collapsed remarkably well into the master curves. Similar results were verified for the neat UHMWPE and the remaining nanocomposites. Hence the methodology developed for the UHMWPE [6] proved to be also effective when applied to the UHMWPE/MWCNT nanocomposites.

The creep tests done at $37^{\circ} \mathrm{C}$ enabled the verification of the viscoelastic model developed solely by using the dynamic experimental data. In Figure 10 are plotted the experimental creep compliances for all the cases. The creep compliance predictions based on the viscoelastic model are also included in Figure 10. The model compares well with the experimental data but tended to deviate at longer times. Since in the present circumstances the model parameters were obtained from a very short-term testing, they are not appropriate for long-term extrapolation. However the viscoelastic behaviour remains unchanged for all nanocomposites, since all creep curves can be superimposed on a unique curve by a vertical shift. It seems, therefore, that the MWCNTs are almost neutral what concerns the viscoelastic behaviour of UHMWPE nanocomposites, at least for the compositions used for analysed during this study. Although the interface quality between the polymer and MWCNTs was better for the HDPE, since large scale polymer relaxations in the HDPE/MWCNT (1.0\%) nanocomposites were effectively restrained by the presence of MWCNTs, the shift factors obtained from the TTSP remain the same irrespective of the presence of carbon nanotubes [10]. This allows concluding that the viscoelastic nature of HDPE and UHMWPE are unaltered by the inclusion of MWCNTs. 
Some concerns may be raised about the assumption of linear viscoelastic behaviour at elevated temperatures. For polymers, in general, the transition from linear to non-linear viscoelastic behaviour happens between 10 and $20 \%$ of the ultimate tensile strength (UTS). In the present case the load levels were always lower than $1 \mathrm{MPa}$, as indicated previously, assuming a UTS of about 20MPa it means a load level lower than 5\% UTS. This is for room temperature $\left(22-23^{\circ} \mathrm{C}\right)$. For higher temperature a drop on the UTS should be expected. According to [12], the UTS at $80^{\circ} \mathrm{C}$ drops to $5 \mathrm{MPa}$. This means that the load level imposed becomes close to $20 \%$ UTS. Therefore at this temperature levels the polymer may experience a transition to the non-linear viscoelastic behaviour at the same load levels. Nevertheless since the TTSP was applicable for all operating temperature range, $22^{\circ} \mathrm{C}-82^{\circ} \mathrm{C}$, we may conclude that the polymer behaviour was always in the linear viscoelastic domain.

\section{Conclusions}

Modeling viscoelastic properties of a neat UHMWPE medical grade and its nanocomposites reinforced with MWCNTs was preformed in the linear range, by means of dynamic testing. The applicability of the Time-Temperature Superposition Principle (TTSP) to the dynamic viscoelastic properties was checked for all cases. The methodology used enabled to determine the horizontal and vertical shift factors for the TTSP. According to the Delay and Plazek definition, these UHMWPE and its nanocomposites may be considered thermorheologically simple, although this behavior can only be assumed for the frequency and temperature ranges used in this study.

Validation was performed using experimental creep data. The viscoelastic models obtained via dynamic viscoelastic properties compared well with experimental data but tended to deviate at longer times.

The unaltered viscoelastic characteristics of the neat UHMWPE and its nanocomposites provide important data in what concerns further modelling or tailoring of the material viscolelastic properties. Moreover, although the conclusion here withdrawn being established for the linear domain, it seems rather natural to extend its validity for the non-linear domain.

\section{Acknowledgements}


The author R. M. Guedes would like to thank the FCT (Portuguese Science and Technology Foundation) for financial support provided under the grant PTDC/EME-PME/114808/2009 for the present study.

\section{References}

[1] Enrique Gomez-Barrena, José-Antonio Puertolas, Luis Munuera and Yrjö T Konttinen. Update on UHMWPE research. From the bench to the bedside. Acta Orthopaedica 2008; 79 (6): 832-840.

[2] Elena Maria Brach del Prever, Alessandro Bistolf, Pierangiola Bracco, Luigi Costa. UHMWPE for arthroplasty: past or future? J Orthopaed Traumatol (2009) 10:1-8.

[3] Yeong-Seok Zoo, Jeong-Wook An, Dong-Phil Lim, and Dae-Soon Lim. Effect of carbon nanotube addition on tribological behavior of UHMWPE. Tribology Letters (2004) 16(4):305-309.

[4] Jeong-Ho Ko, Jin-Hae Chang. Properties of Ultrahigh-Molecular-Weight Polyethylene Nanocomposite Films Containing Different Functionalized Multiwalled Carbon Nanotubes. Polymer Engineering and Science (2009) 49:2168-2178.

[5] A. Fonseca, N. Inácio, S. Kanagaraj, M. S. A. Oliveira, and J. A. O. Simões. The Use of Taguchi Technique to Optimize the Compression Moulding Cycle to Process Acetabular Cup Components. Journal of Nanoscience and Nanotechnology (2011) 11:1-6.

[6] R.M. Guedes, A viscoelastic model for a biomedical ultra-high molecular weight polyethylene using the time-temperature superposition principle, Polymer Testing 30 (2011) 294-302.

[7] Dealy J., Plazek D. Time-temperature superposition—a users guide. Rheol. Bull. 78 (2):16-31, 2009.

[8] Heymans Nicole, Constitutive equations for polymer viscoelasticity derived from hierarchical models in cases of failure of time-temperature superposition, Signal Processing 83 (2003) $2345-2357$.

[9] Findley, W.N., Lai, J.S. and Onaran, K., Creep and Relaxation of Nonlinear Viscoelastic Materials, Dover Publications, New York, 1989, 239-248.

[10] S. Kanagaraj, R. M. Guedes, Mónica S. A. Oliveira, and José A. O. Simões. Dynamic Mechanical Analysis of Multi-Walled Carbon Nanotube/HDPE Composites. J. Nanosci. Nanotechnol. 8 (2008) 4008-4012. 
[11] S. Affatato. Ageing of polyethylene composite implants in medical devices. In Ageing of composites, edited by R Martin. Woodhead Publishing Limited, Cambridge 2011.

[12] Ticona 2001. Ticona GUR® (PE-UHMW) Product Brochure. Downloaded online at: http://www.hipolymers.com.ar/pdfs/gur/diseno/GUR\%20\%28PE-UHMW\%29.pdf. 


\section{List of Figures}

Figure 1: Storage compliance measured from $0.1 \mathrm{~Hz}$ to $50 \mathrm{~Hz}$ at $37^{\circ} \mathrm{C}$ for the neat UHMWPE and UHMWPE/MWCNT (1.0\%).

Figure 2: Storage compliance measured from 22 to $87^{\circ} \mathrm{C}$ at $1 \mathrm{~Hz}$ for the neat UHMWPE and UHMWPE/MWCNT (1.0\%).

Figure 3: Loss compliance measured from $0.1 \mathrm{~Hz}$ to $50 \mathrm{~Hz}$ at $37^{\circ} \mathrm{C}$ for the neat UHMWPE and UHMWPE/MWCNT (1.0\%).

Figure 4: Loss compliance measured from 22 to $87^{\circ} \mathrm{C}$ at $1 \mathrm{~Hz}$ for the neat UHMWPE and UHMWPE/MWCNT (1.0\%).

Figure 5: Temperature dependency of model exponent $\alpha$ for neat UHMWPE and UHMWPE/MWCNT (1.0\%).

Figure 6: Averaged exponent $\alpha$ for neat UHMWPE and UHMWPE/MWCNT nanocomposites.

Figure 7: Model parameters $\mathrm{S}_{0}$ and $\mathrm{S}_{1}$ for neat UHMWPE and UHMWPE/MWCNT nanocomposites.

Figure 8: Arrhenius activation energies $\Delta \mathrm{H}_{\alpha}$ and $\Delta \mathrm{H}_{\beta}$ for horizontal and vertical shift factors, respectively, versus MWCNT weight fraction.

Figure 9: Master curves for storage and loss compliance for UHMWPE/MWCNT (1.0\%).

Figure 10: Creep compliance measured for neat UHMWPE and UHMWPE/MWCNT nanocomposites

Figure 11: Measured creep compliance compared against model prediction, obtained from DMTA, for UHMWPE/MWCNT (1.0\%). 
Figure 12: Experimental creep compliance for neat UHMWPE and UHMWPE/MWCNT nanocomposites obtained for the first hundred seconds.

Figure 13: Predicted creep compliance for neat UHMWPE and UHMWPE/MWCNT nanocomposites for the first hundred seconds. 


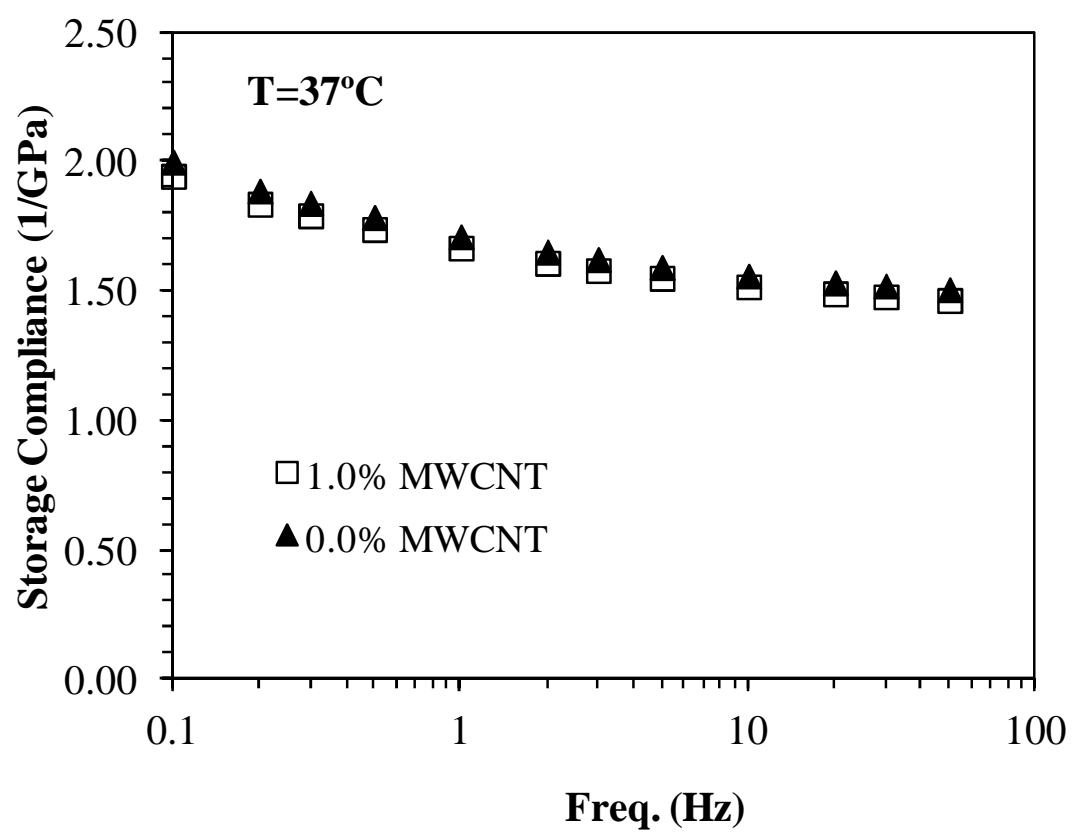

Figure 1: Storage compliance measured from $0.1 \mathrm{~Hz}$ to $50 \mathrm{~Hz}$ at $37^{\circ} \mathrm{C}$ for the neat UHMWPE and UHMWPE/MWCNT (1.0\%).

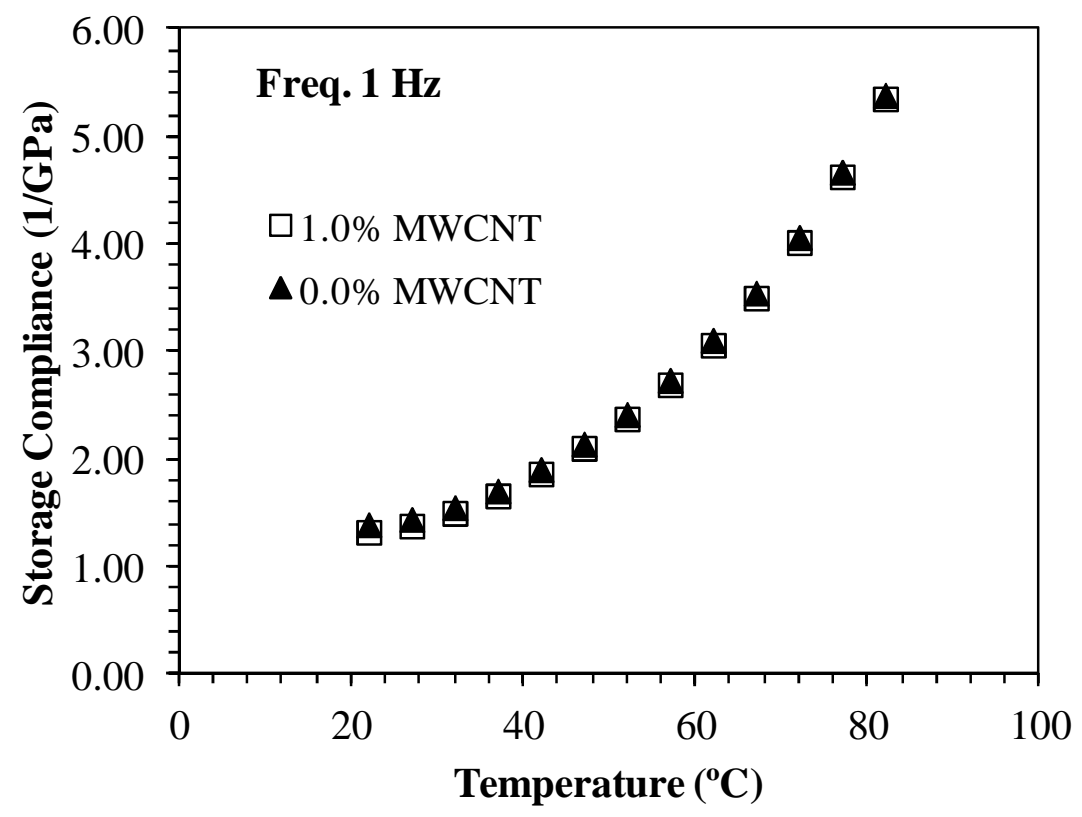

Figure 2: Storage compliance measured from 22 to $87^{\circ} \mathrm{C}$ at $1 \mathrm{~Hz}$ for the neat UHMWPE and UHMWPE/MWCNT (1.0\%). 


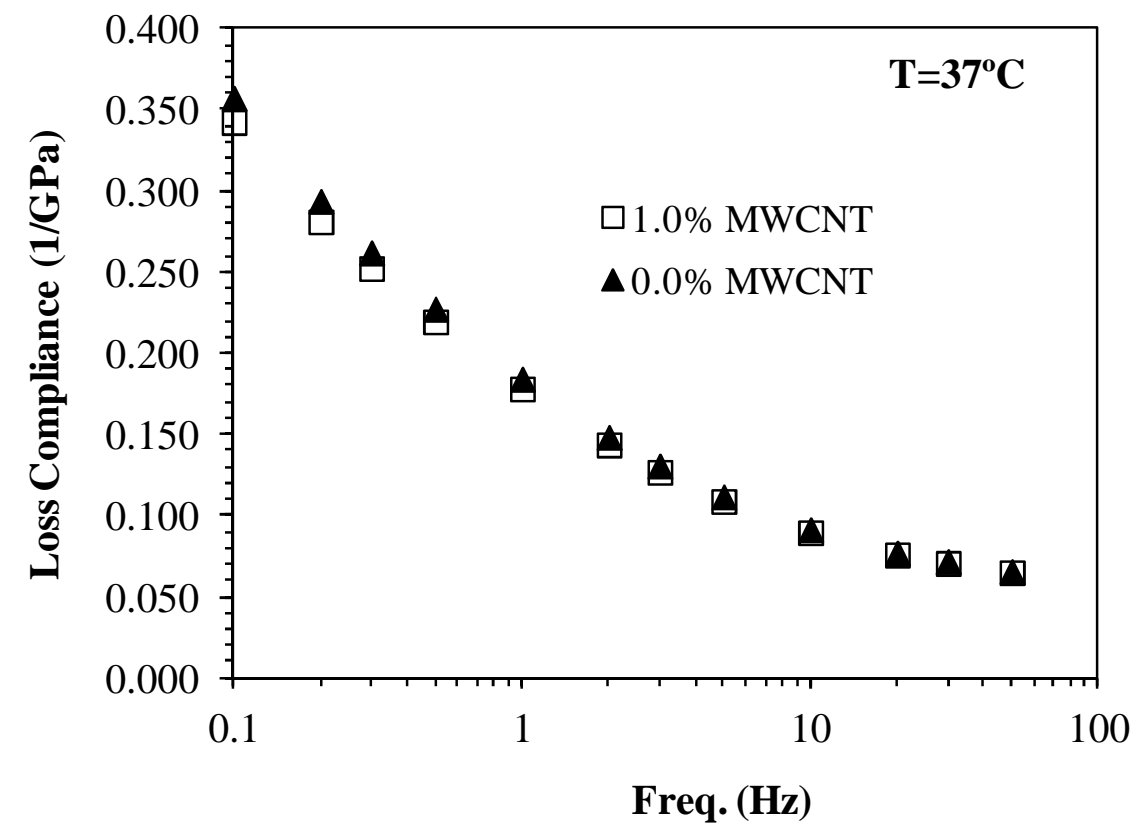

Figure 3: Loss compliance measured from $0.1 \mathrm{~Hz}$ to $50 \mathrm{~Hz}$ at $37^{\circ} \mathrm{C}$ for the neat UHMWPE and UHMWPE/MWCNT (1.0\%).

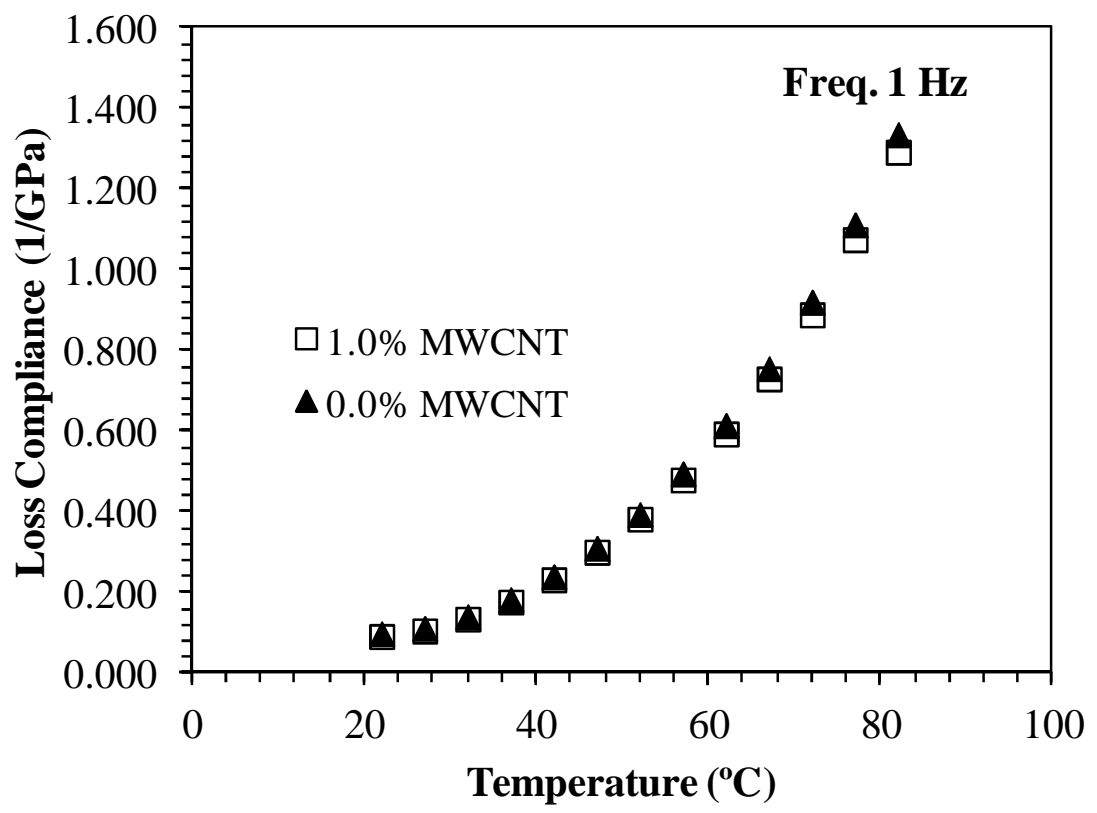

Figure 4: Loss compliance measured from 22 to $87^{\circ} \mathrm{C}$ at $1 \mathrm{~Hz}$ for the neat UHMWPE and UHMWPE/MWCNT (1.0\%). 


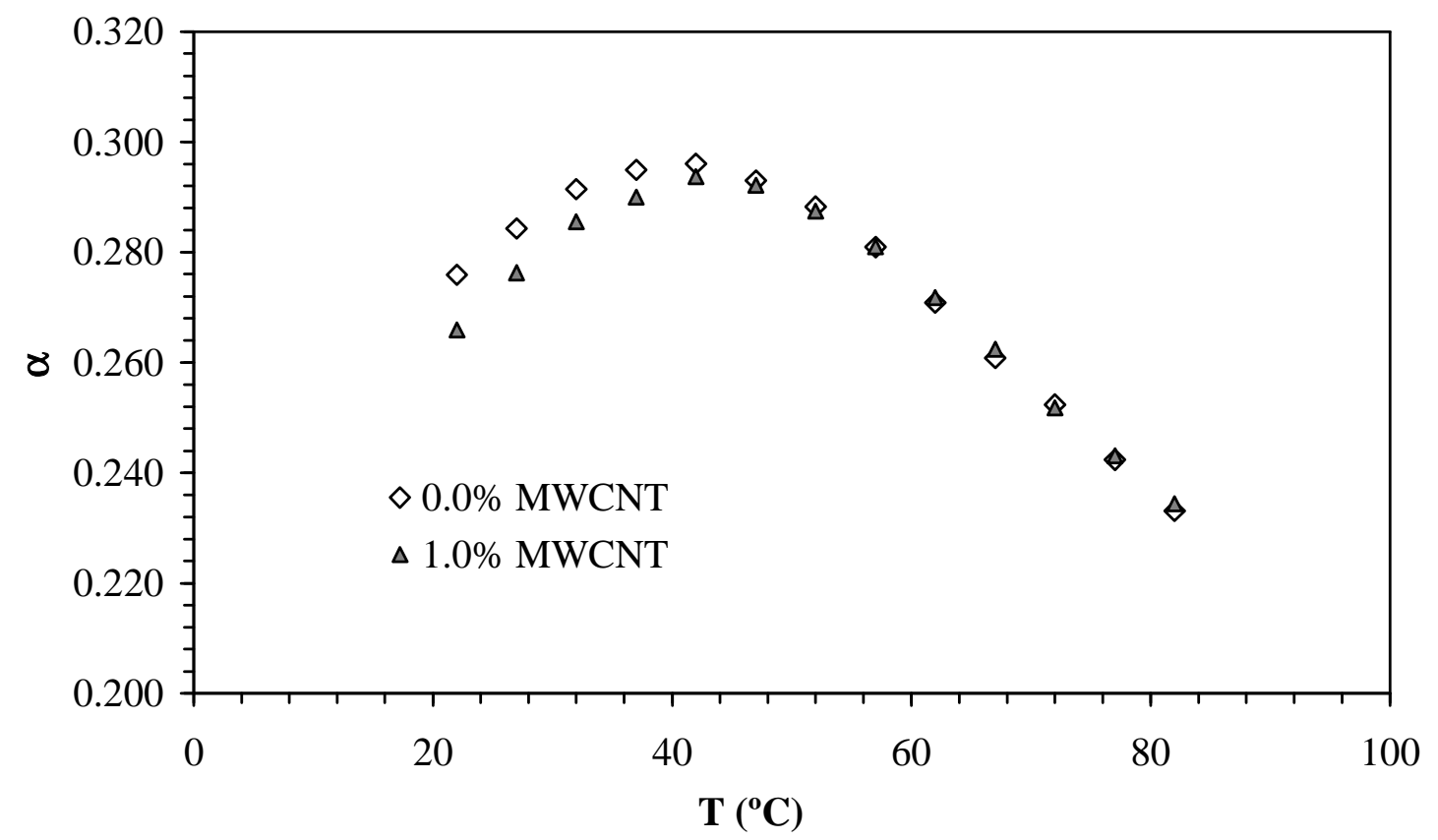

Figure 5: Temperature dependency of model exponent $\alpha$ for neat UHMWPE and UHMWPE/MWCNT (1.0\%).

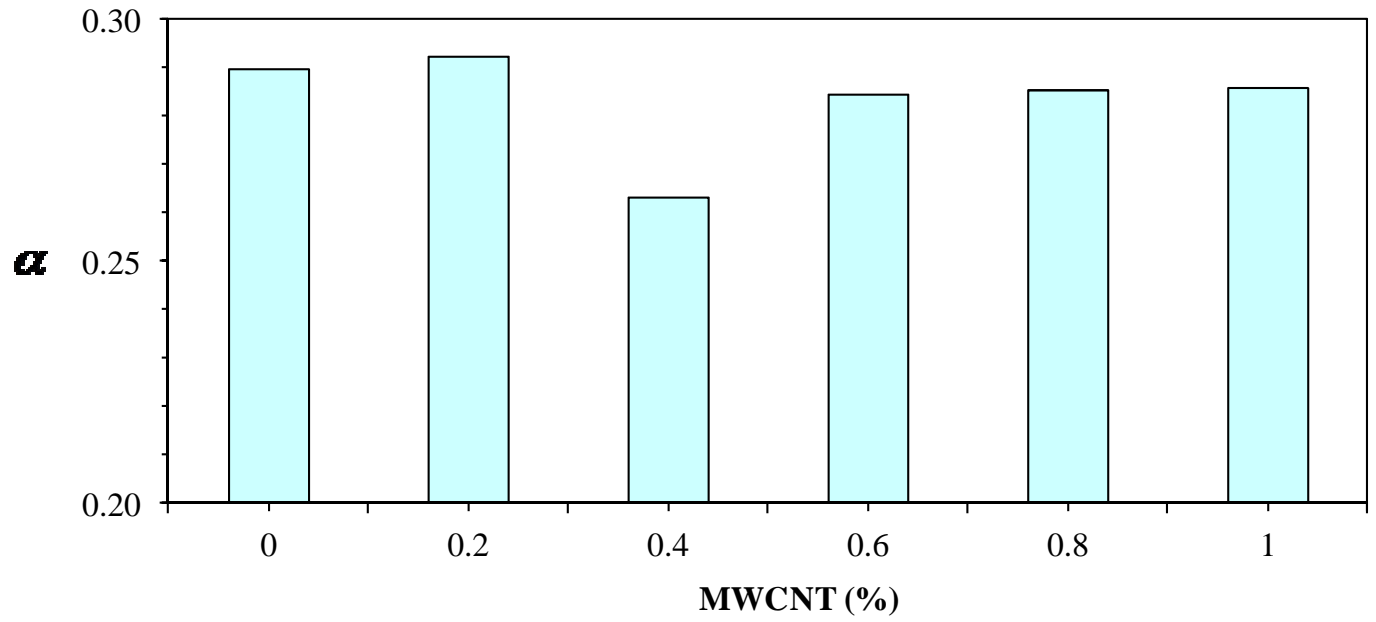

Figure 6: Averaged exponent $\alpha$ for neat UHMWPE and UHMWPE/MWCNT nanocomposites. 


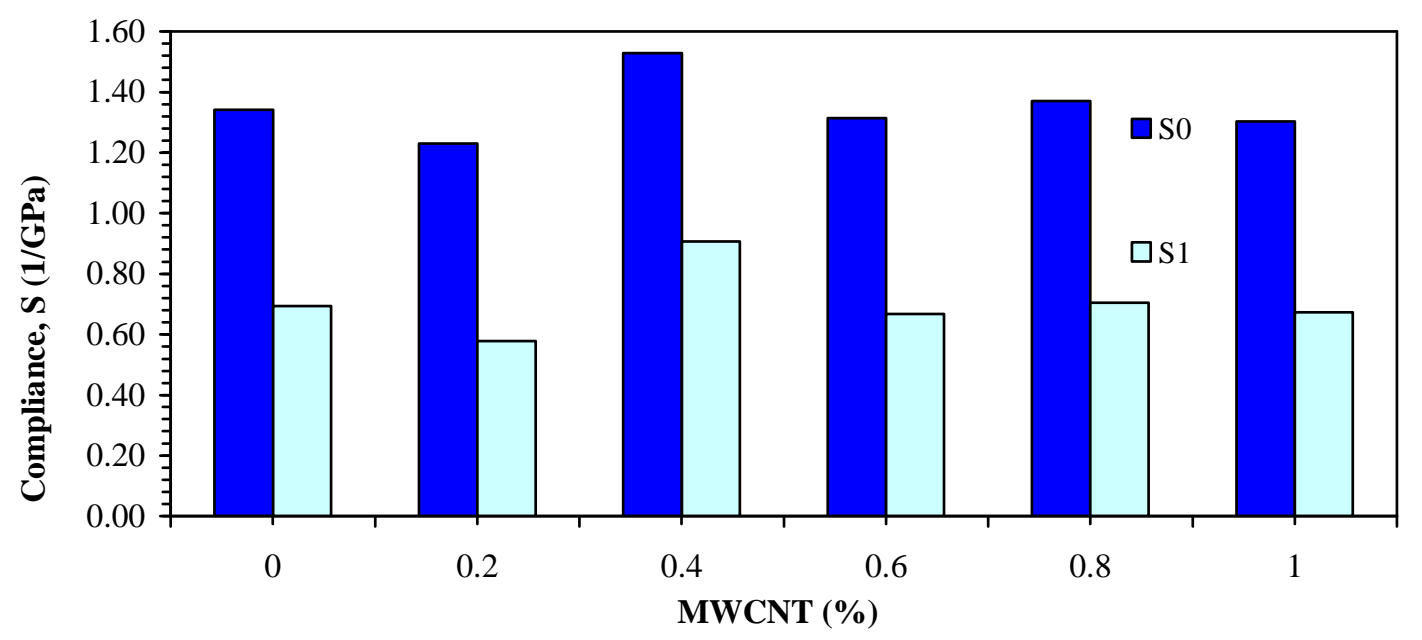

Figure 7: Model parameters $\mathrm{S}_{0}$ and $\mathrm{S}_{1}$ for neat UHMWPE and UHMWPE/MWCNT nanocomposites.

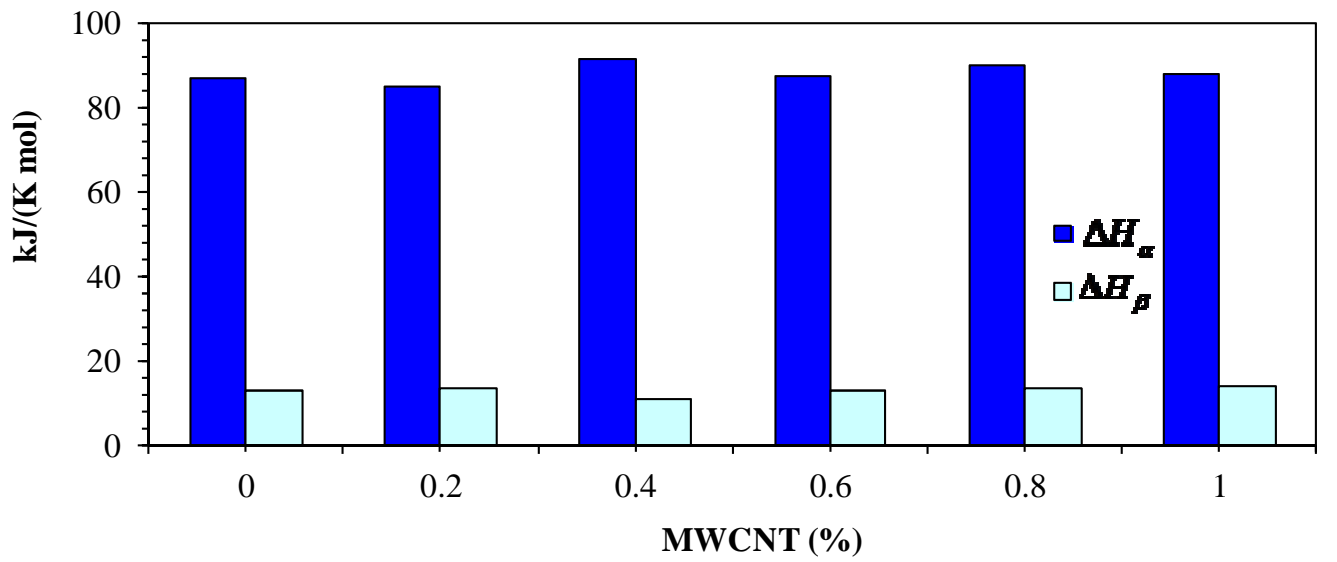

Figure 8: Arrhenius activation energies $\Delta \mathrm{H}_{\alpha}$ and $\Delta \mathrm{H}_{\beta}$ for horizontal and vertical shift factors, respectively, versus MWCNT weight fraction. 

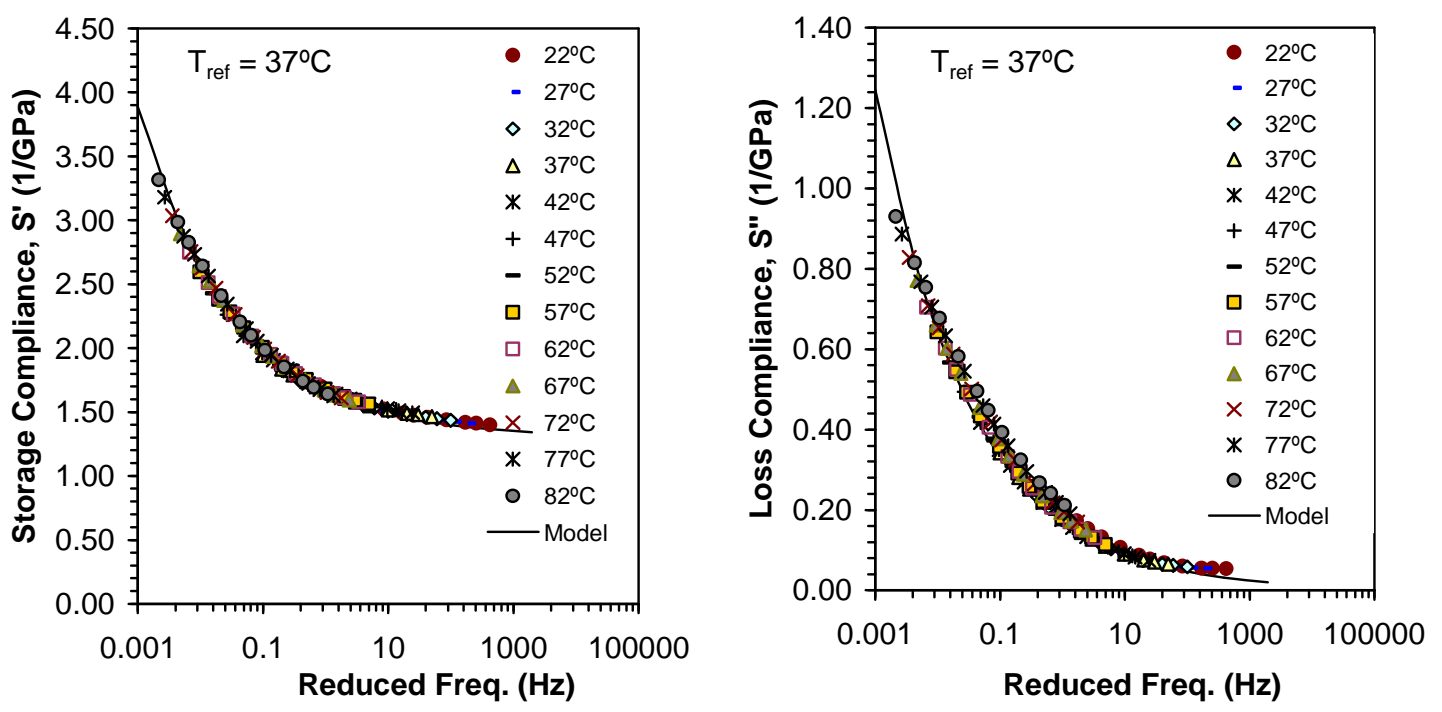

Figure 9: Master curves for storage and loss compliance for UHMWPE/MWCNT (1.0\%).

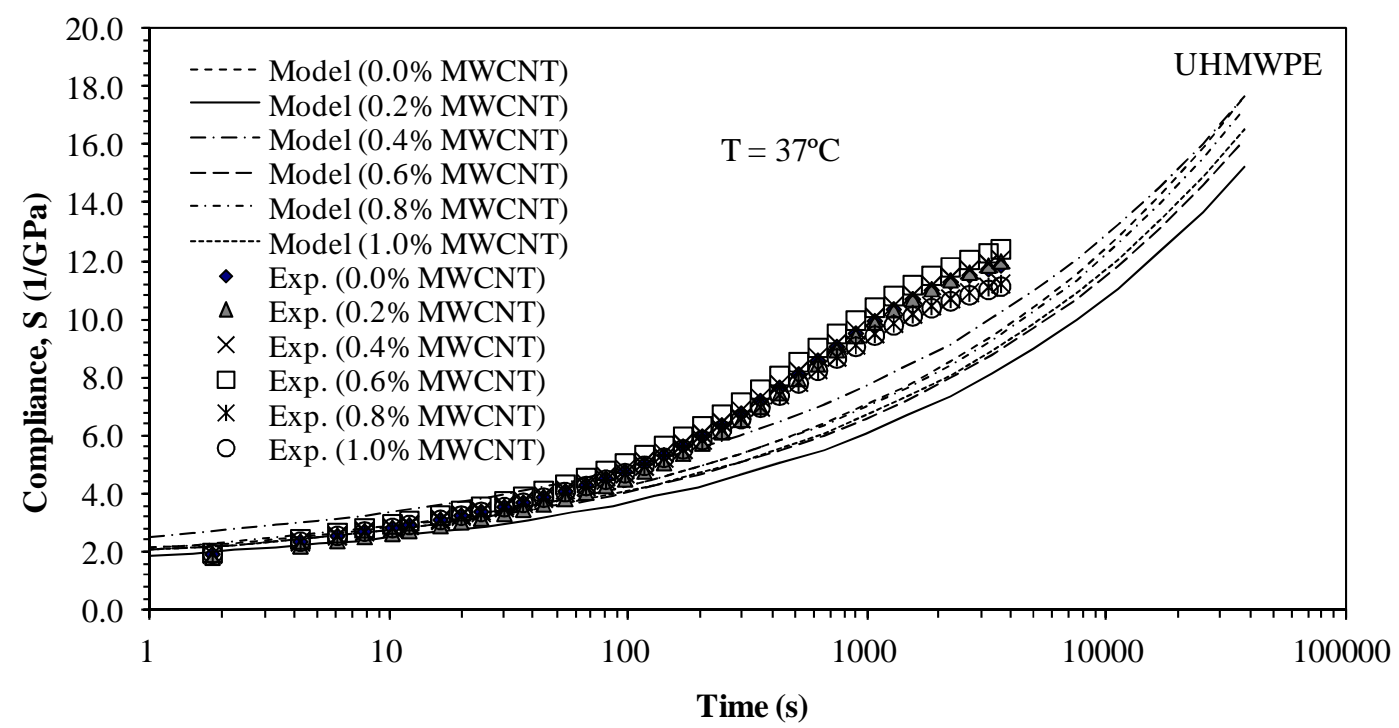

Figure 10: Creep compliance measured for neat UHMWPE and UHMWPE/MWCNT nanocomposites compared against model prediction, obtained from DMTA. 